\title{
Bulk and interfacial properties of binary hard-platelet fluids
}

\author{
M. Bier $⿴$ L. Harnau, and S. Dietrich \\ Max-Planck-Institut für Metallforschung, Heisenbergstraße 3, D-70569 Stuttgart, Germany, and \\ Institut für Theoretische und Angewandte Physik, Universität Stuttgart, \\ Pfaffenwaldring 57, D-70569 Stuttgart, Germany
}

(Dated: October 30, 2003)

\begin{abstract}
Interfaces between demixed fluid phases of binary mixtures of hard platelets are investigated using density-functional theory. The corresponding excess free energy functional is calculated within a fundamental measure theory adapted to the Zwanzig model, in which the orientations of the particles of rectangular shape are restricted to three orthogonal orientations. Density and orientational order parameter profiles at interfaces between coexisting phases as well as the interfacial tension are determined. A density inversion, oscillatory density profiles, and a Fisher-Widom line have been found in a mixture of large thin and small thick platelets. The lowest interfacial tension corresponds to the mean bulk orientation of the platelets being parallel to the interface. For a mixture of large and small thin platelets, complete wetting of an isotropic-nematic interface by a second nematic phase is found.
\end{abstract}

PACS numbers: 61.20.Gy, 61.30.Hn, 68.05.-n, 82.70.Dd

\section{INTRODUCTION}

There is growing interest in thermal and structural properties of suspensions of platelike colloids like blood, clay sols, or liquid crystal dispersions because of possible applications in biomedicine (e.g. shape-selective separation of cell components [1]), geophysics (e.g. oil drilling [2]), or liquid crystal display technology [3]. From theoretical considerations [4, 5] and computer simulations [6, [7, [8] one expects an isotropic to nematic transition due to the orientational degrees of freedom for sufficiently high platelet concentrations. This transition is not observed for clays because the long-ranged Coulomb interaction between the charged clay particles leads to gelation [9, 10]. Nonetheless, recent preparation methods have been developed to produce suspensions of sterically stabilized platelets which do exhibit the expected isotropic to nematic transition 11, 12, 13, 14]. It was shown experimentally 13 and theoretically 15, 16, 17, 18] that polydispersity in the size of the platelets strongly affects the phase behavior. In particular, binary mixtures of thin and thick platelets lead to an unexpected isotropic-nematic density inversion 13, 16. Although attention has been paid to the bulk phase behavior 17, 18], so far there are no studies on fluid-fluid interfacial properties of such mixtures. On the basis of recent theoretical studies on fluids of thin hard platelets near hard walls [19, 20], we expect that the competition of orientational entropy and excluded volume interaction leads to interesting fluid-fluid interfacial properties which should be experimentally accessible via optical techniques.

In this paper, we study interfacial properties between coexisting isotropic and nematic phases of a binary mixture of hard square cuboids within the Zwanzig approx- imation 21]. This model is chosen as the simplest nontrivial approximation to the above-mentioned properties of real platelet suspensions. Binary mixtures are used to mimic polydispersity. The hard-particle approximation is doubtful for long-ranged platelet-platelet interactions, but there are model systems of quasi-hard platelets 11, 12]. The actual shape of the platelets (disks, stripes, hexagons, or square cuboids) is expected to be less important, only the thickness $L$ and the width $D$ are assumed to be relevant. Finally, the square face of the platelets can take only three rather than a continuous range of orientations (Zwanzig model 21]). This model offers the advantage that the excess free energy functional can be determined within a fundamental measure theory 22, 23, 24, 25] and that the difficult determination of inhomogeneous density profiles becomes numerically feasible allowing one to study interfacial properties of binary hard-platelet fluids in detail. Due to the approximations, we only expect to find qualitatively correct results.

This paper is organized as follows. In Sec. I we describe the density functional and the fundamental measure theory. Sec. III] presents two representative bulk phase diagrams of binary hard-platelet mixtures involving isotropic, nematic, and columnar phases. In Sec. IV] we determine the density and the orientational order parameter profiles as well as the interfacial tensions of isotropic-nematic interfaces in a mixture of large thin and small thick platelets. Sec. $\nabla$ presents density profiles at nematic-nematic interfaces. The isotropic-nematic interfaces near an isotropic-nematic-nematic triple point are investigated in Sec. VI] Our results are summarized in Sec. VII

*Electronic address: bier@fluids.mpi-stuttgart.mpg.de 


\section{DENSITY FUNCTIONAL AND FUNDAMENTAL MEASURE THEORY}

We consider a binary mixture of hard rectangular particles of size $L_{i} \times D_{i} \times D_{i}, i \in\{1,2\}$. The position of the center of mass $\mathbf{r}$ is a continuous variable, whereas the normal of the square face is restricted to directions $\beta \in\{x, y, z\}$. The number density of the centers of mass of the platelets of size $i$ and orientation $\beta$ at position $\mathbf{r}$ is denoted by $\varrho_{i, \beta}(\mathbf{r})$. In the absence of external potentials, the equilibrium density profiles of the mixture minimize the grand potential functional

$$
\begin{aligned}
\Omega\left[\left\{\varrho_{i, \beta}\right\}\right]= & \sum_{i, \beta} \int \mathrm{d}^{3} r \varrho_{i, \beta}(\mathbf{r})\left(k_{B} T\left(\ln \left(\varrho_{i, \beta}(\mathbf{r}) \Lambda_{i}^{3}\right)-1\right)\right. \\
& \left.-\mu_{i}\right)+F^{\operatorname{ex}}\left[\left\{\varrho_{i, \beta}\right\}\right]
\end{aligned}
$$

where the chemical potential $\mu_{i}$ and the thermal de Broglie wavelength $\Lambda_{i}$ of platelets of size $i$ are independent of the particle orientation.

In fundamental measure theory, one postulates the following form of the excess free energy functional [22, 23, 24, 25]:

$$
F^{\mathrm{ex}}\left[\left\{\varrho_{i, \beta}\right\}\right]=k_{B} T \int \mathrm{d}^{3} r \Phi\left(\left\{n_{l}(\mathbf{r})\right\}\right)
$$

with the reduced excess free energy density

$$
\begin{aligned}
\Phi\left(\left\{n_{l}\right\}\right)= & -n_{0} \ln \left(1-n_{3}\right)+\sum_{\sigma \in\{x, y, z\}} \frac{n_{1 \sigma} n_{2 \sigma}}{1-n_{3}} \\
& +\frac{n_{2 x} n_{2 y} n_{2 z}}{\left(1-n_{3}\right)^{2}}
\end{aligned}
$$

and the weighted densities

$$
n_{l}(\mathbf{r})=\sum_{i, \beta} \int \mathrm{d}^{3} r^{\prime} \omega_{i, \beta}^{(l)}\left(\mathbf{r}-\mathbf{r}^{\prime}\right) \varrho_{i, \beta}\left(\mathbf{r}^{\prime}\right)
$$

for $l \in\{0,1 x, 1 y, 1 z, 2 x, 2 y, 2 z, 3\}$.

The weight functions $\omega_{i, \beta}^{(l)}$ are obtained by expressing the Fourier transform of the Mayer- $f$ function as a sum of products of single particle functions. The Mayer- $f$ function equals -1 if the particles overlap and is zero otherwise. Explicit expressions of the weight functions are documented in Refs. [22, 23, 24, 25]. In the limit of infinitely thin platelets $\left(L_{i} / D_{i} \rightarrow 0\right)$ the excess free energy density reduces to a third-order virial approximation [20]. For convenience, we introduce the dimensionless variables $\mu_{i}^{*}:=\mu_{i} / k_{B} T+3 \ln \left(D_{1} / \Lambda_{i}\right)$.

\section{BULK PHASE DIAGRAMS}

The bulk phase behavior of spatially homogeneous binary platelet fluids has recently been studied within the
Zwanzig model [17]. Rich phase diagrams are found involving an isotropic and one or two nematic phases characterized by different concentrations. The phase diagrams are very sensitive to the size ratio $D_{1} / D_{2}$ and to the aspect ratios $L_{1} / D_{1}$ and $L_{2} / D_{2}$.

Fig. 1 shows two representative examples of bulk phase
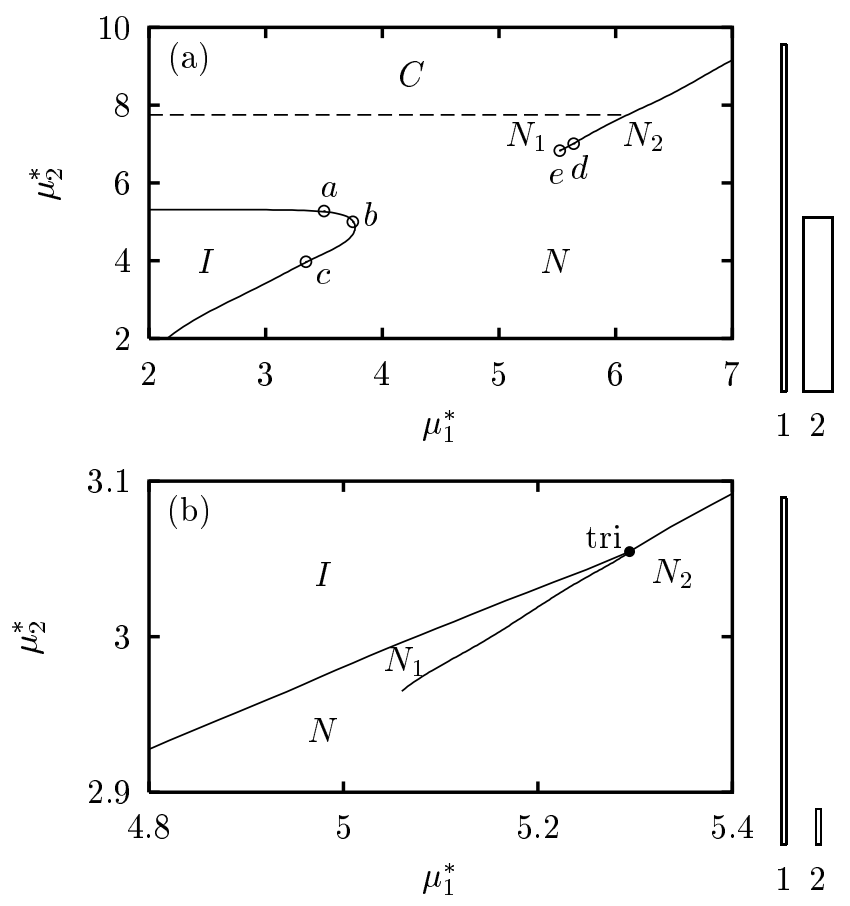

FIG. 1: Bulk phase diagrams of binary mixtures of large and small hard platelets $\left(L_{1} / D_{1}=0.01 ;\right.$ (a): $D_{1} / D_{2}=2$, $L_{2} / D_{2}=0.16$; (b): $D_{1} / D_{2}=10, L_{2} / D_{2}=0.1$; see also the schematic side views $L_{i} \times D_{i}$ on the right side bar) in terms of the reduced chemical potentials $\mu_{1}^{*}$ and $\mu_{2}^{*}$. Full lines represent strongly first-order transitions, whereas dashed lines denote second-order or weakly first-order transitions. In (a), one isotropic phase $I$, one nematic phase $N$, and one columnar phase $C$ are found. The isotropic-nematic transitions in the monodisperse limits occur at $\mu_{1}^{*}=0.829(1)$ for the large platelets $\left(\mu_{2}^{*} \rightarrow-\infty\right)$ and at $\mu_{2}^{*}=5.505(1)$ for the small platelets $\left(\mu_{1}^{*} \rightarrow-\infty\right)$. Density and order parameter profiles for isotropic-nematic interfaces at state points $a, b$, and $c$ are shown in Fig. 2 The nematic phase $N$ demixes into two nematic phases $N_{1}$ and $N_{2}$. Fig. 4 exhibits density profiles for nematic-nematic interfaces at state points $d$ and $e$, close to the lower critical point of the $N_{1}-N_{2}$ demixing. For small values of $\mu_{1}^{*}$, the nematic-columnar transition is of second or weakly first order (dashed line), whereas it is strongly firstorder (solid line) if $\mu_{1}^{*}$ is sufficiently large. The dashed line appears to be straight because for this range of the chemical potentials, the density of the large platelets is negligible compared to that of the small platelets. In (b), only a small part of the phase diagram around the triple point tri is shown, where the isotropic phase $I$ coexists with two nematic phases $N_{1}$ and $N_{2}$.

diagrams of binary mixtures of large and small platelets in terms of the reduced chemical potentials $\mu_{1}^{*}$ and $\mu_{2}^{*}$. The coexistence curves in these diagrams are calculated 
numerically by finding pairs of equilibrium states with equal chemical potentials and pressures. The phase transitions at full curves are strongly first-order, whereas dashed lines denote second-order or weakly first-order transitions.

For a binary fluid consisting of large thin and small thick platelets (see Fig. 1(a)), a single nematic phase $N$ is found, which is separated from the isotropic phase $I$ by a first-order phase boundary line. At larger values of the two chemical potentials, the nematic phase $N$ demixes discontinuously into two nematic phases $N_{1}$ and $N_{2}$. Furthermore, the nematic phase $N$ undergoes a phase transition into a columnar phase $C$, where parallel columns of small platelets are surrounded by single large platelets with orientations perpendicular to the column axes. For small values of $\mu_{1}^{*}$, the nematic-columnar transition is of second or weakly first order. The phase boundary line appears straight because the density of the large platelets is negligible there, so that the mixture is nearly monodisperse. The nematic-columnar boundary line changes to strongly first-order at the tricritical point where the nematic-nematic boundary line ends.

Upon decreasing the size of the small platelets, the lower critical point of this first-order $N_{1}-N_{2}$ coexistence curve shifts to smaller values of the chemical potentials until the $N_{1}-N_{2}$ and the $I-N$ coexistence curves start to intersect, giving rise to a triple point at which two nematic phases $N_{1}$ and $N_{2}$ coexist with the isotropic phase $I$ (see Fig. प(b)).

\section{ISOTROPIC-NEMATIC INTERFACE}

In this section we discuss the properties of interfaces between the coexisting isotropic and nematic phases depicted in the phase diagram of Fig. 1(a). The interface normal is always arranged to point in $z$-direction. The results are expressed in terms of the orientationally averaged number density

$$
\varrho_{i}(z):=\varrho_{i, x}(z)+\varrho_{i, y}(z)+\varrho_{i, z}(z),
$$

the nematic order parameter

$$
s_{i}(z):=\frac{\varrho_{i, z}(z)-\frac{1}{2}\left(\varrho_{i, x}(z)+\varrho_{i, y}(z)\right)}{\varrho_{i}(z)},
$$

and the biaxial order parameter

$$
q_{i}(z):=\frac{\varrho_{i, x}(z)-\varrho_{i, y}(z)}{\varrho_{i}(z)} .
$$

Fig. 2 shows the density and the nematic order parameter profiles of the small thick platelets for the state points denoted as $a, b$, and $c$ in Fig. 1(a). The interface normal and the director of the nematic phase are parallel and the position $z=0$ of the origin is fixed by choosing $s_{1}(0)=\frac{1}{2}$. No biaxiality has been observed, that is, $q_{i}(z) \equiv 0$. We have confirmed that the density profiles
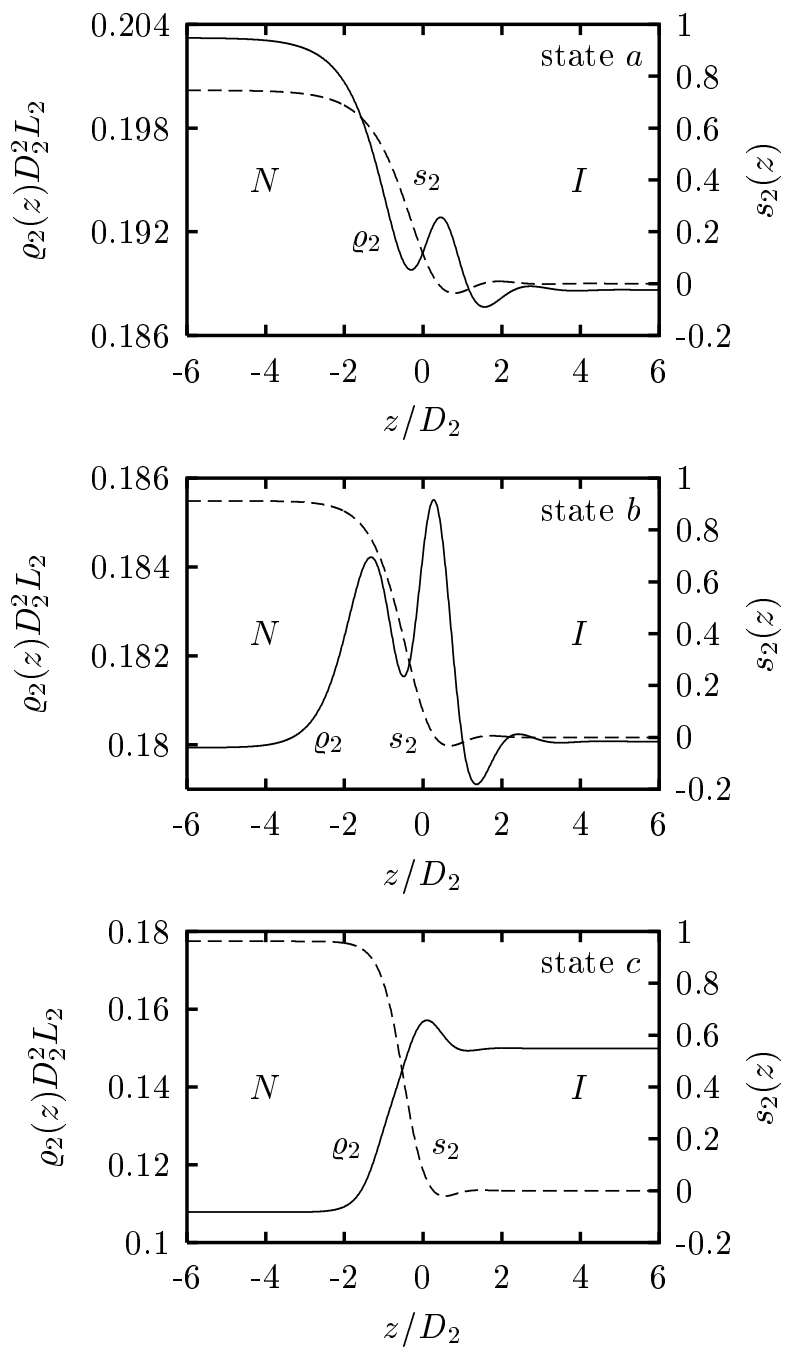

FIG. 2: Orientationally averaged density profiles $\varrho_{2}$ (solid lines) and nematic order parameter profiles $s_{2}$ (dashed lines) of the small thick platelets for the state points $a, b$, and $c$ in Fig. 1(a) at a planar interface between the coexisting isotropic phase $I$ at $z \rightarrow \infty$ and the nematic phase $N$ at $z \rightarrow-\infty$. The position $z=0$ of the origin is chosen to be given by $s_{2}(0)=\frac{1}{2}$. The interface normal and the director of the nematic phase are parallel, which corresponds to the lowest surface tension (see Fig. 3). A density inversion occurs near the state point $b$. At the interface layers form which slightly prefer platelet orientations perpendicular to the interface normal.

for both thin and thick platelets show oscillations of the same period, but the amplitude for the thin platelets is considerably smaller than for the thick platelets.

Whereas the density of the thin platelets is always smaller in the isotropic phase than in the nematic phase along the coexistence curve, a density inversion for the thick platelets is found. For state $b$, the density of the small thick platelets in the isotropic and the nematic phase are nearly identical. This remarkable phenomenon of isotropic-nematic density inversion has been observed experimentally for a dispersion of sterically stabilized 
gibbsite $\left(\mathrm{Al}(\mathrm{OH})_{3}\right)$ platelets 13 .

For isotropic-anisotropic coexistence in binary mixtures of long and short thin rods, one observes a large fraction of long rods in the anisotropic phase if there is a small fraction of the long rods in the isotropic phase [26]. For platelets this fractionation does not occur to the same extend because the density $\varrho_{1}$ increases by at most one order of magnitude upon traversing the transition from the isotropic to the nematic phase.

The nematic order parameter profiles shown in Fig. 2 exhibit a small negative minimum $\left(s_{2}(z)<0\right)$ on the isotropic side of the interface implying a depletion of platelets with orientation parallel to the interface. A similar minimum has been found for freely rotating platelets near a hard wall 19]. This indicates that the nematic phase acts like a wall creating a corresponding depletion zone in the adjacent isotropic phase.

The surface tension $\gamma_{I N}$ of an isotropic-nematic interface with the director of the nematic phase at $z \rightarrow-\infty$ parallel $(\|)$ or perpendicular $(\perp)$ to the interface normal is shown in Fig. 3 as a function of the pressure $p$

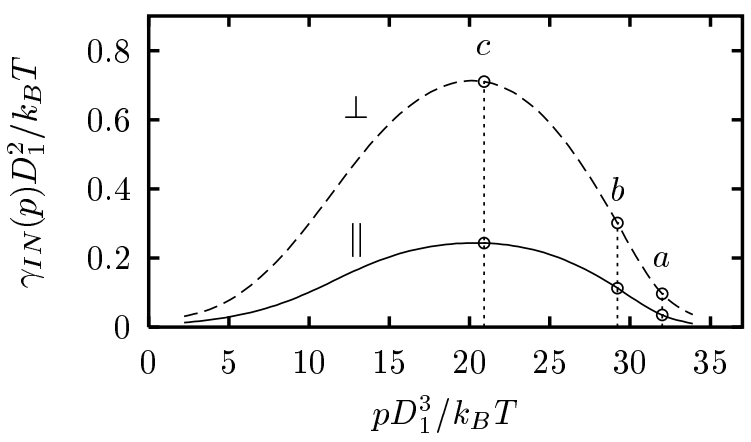

FIG. 3: Isotropic-nematic interfacial tension $\gamma_{I N}$ of a binary mixture of large thin and small thick platelets (see Fig. 1(a)) as a function of the pressure $p$ along the line of phase coexistence. Through the choice of the boundary condition for the orientation of the nematic order at $z \rightarrow-\infty$ the director of the nematic phase can be parallel $(\|)$ or perpendicular $(\perp)$ to the interface normal. The monodisperse fluids, consisting of either the large or the small platelets, correspond to the endpoints of the curves at the left and the right, respectively. The density profiles and the orientational order parameter profiles for the thermodynamic states marked $a, b$ and $c$ are shown in Fig. 2

along the line of coexisting phases. For all pressures, the parallel configuration has the lower surface tension by a factor of rather precisely three. As is apparent from Fig. 3] the surface tension is a non-monotonic function of the pressure and exhibits a maximum close to state $c$. The maximum value of $\gamma_{I N}$ is about 20 times larger than the ones for the monodisperse fluids corresponding to the endpoints of the curves. This implies that the interface of the binary fluid is rather stiff as compared to the ones of the corresponding monodisperse fluids.

Calculating the typical surface tension at the inversion point $b\left(\gamma_{I N}^{\|} D_{1}^{2} / k_{B} T \approx 0.1\right)$ for the above mentioned sterically stabilized gibbsite platelets $\left(D_{1} \approx 170 \mathrm{~nm}\right)$ at room temperature $T=300 \mathrm{~K}$ yields $\gamma_{I N}^{\|} \approx 14 \mathrm{nN} / \mathrm{m}$, which perfectly agrees with the estimate provided in Ref. 13].

A similar behavior has been found for binary mixtures of thin and thick rods [27].

\section{NEMATIC-NEMATIC INTERFACES}

We now turn our attention to the properties of interfaces between two coexisting nematic phases whose corresponding phase boundaries are depicted in the phase diagram of Fig. 1(a). Fig. 4 shows the density pro-
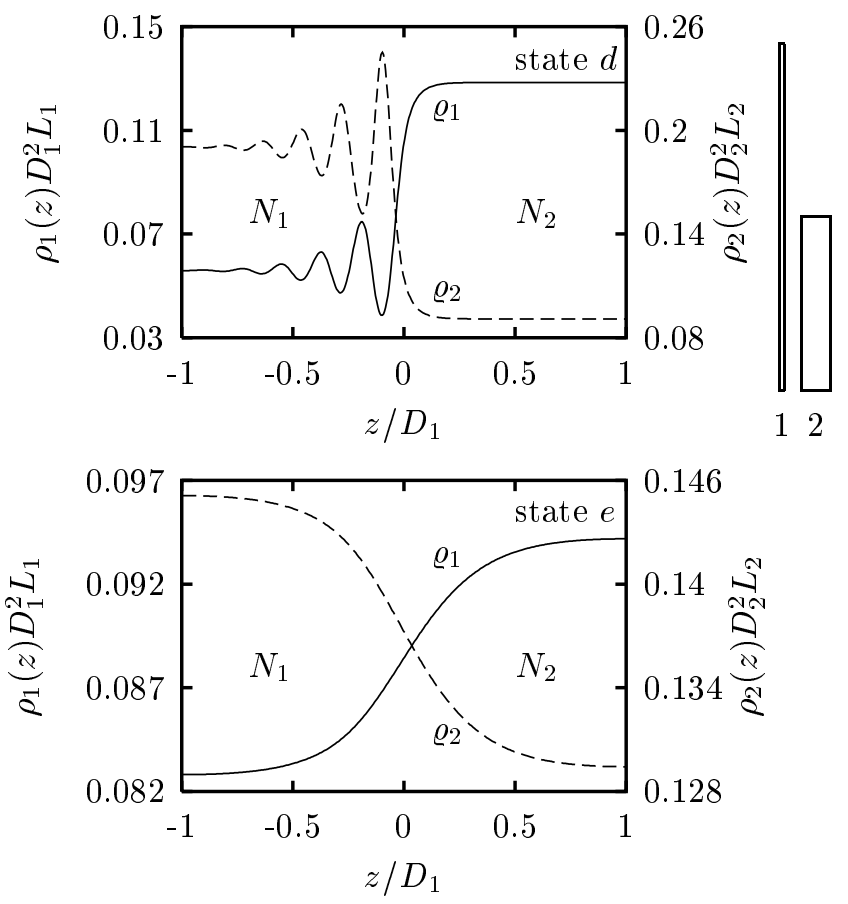

FIG. 4: The density profiles $\varrho_{1}$ (solid lines) and $\varrho_{2}$ (dashed lines) of the large thin and small thick platelets of the binary hard-platelet mixture, respectively, at the planar interface between two coexisting nematic phases $\left(N_{1}\right.$ and $\left.N_{2}\right)$ for the states $d$ and $e$ indicated in Fig. 1(a).

files of the large and small platelets for such interfaces. Pronounced oscillations are found for the state $d$ (see Fig. 1(a)) on that side of the interface which is rich in the small thick platelets. The wave length of the oscillations $\lambda=0.175(5) D_{1}$ is more than twice the thickness of the thick platelets. The exponential decay length $\xi=0.131(4) D_{1}$ is of the same order of magnitude. The wave length $\lambda$ and the exponential decay length $\xi$ are the same for the large and the small platelets.

For the state $e$, closer to the lower critical point of nematic-nematic coexistence, there is no sign of oscillations on either side of the interface. Upon approaching the lower critical point along the nematic-nematic coexistence curve, the density profiles $\varrho_{1}$ and $\varrho_{2}$ turn into 
monotonic functions of $z$ and the interfacial width broadens. In simple fluids, the disappearance of oscillations in the density profiles is connected to the existence of the so-called Fisher-Widom line [28], which divides the bulk phase diagram into regions where the asymptotic decay of bulk two-point correlation function is either monotonic or exponentially damped oscillatory [29, 30]; this behavior of the two-point correlation function carries over to the density profiles. Recently, such Fisher-Widom lines have been found for a binary Gaussian core model 31] and a model colloid-polymer mixture 32]. For the binary platelet model under consideration, the determination of the Fisher-Widom line requires the solution of Ornstein-Zernike equations for a six-component system, two species and three allowed orientations, which is beyond the scope of the present study. However, from the observed vanishing of the oscillations of the density profiles we conclude that there is a Fisher-Widom line and it intersects the coexistence curve between $d$ and $e$.

We have also calculated the surface tensions of nematic-nematic interfaces for different relative orientations of the platelets and the interface. Again the smallest value is taken by the configuration with the mean bulk orientation of the platelets being parallel to the interface (compare Fig. 3). Upon approaching the lower critical point of nematic-nematic coexistence, the expected mean-field-like vanishing of the surface tension and of the $N_{1}-N_{2}$ density difference has been recovered.

\section{ISOTROPIC-NEMATIC INTERFACE NEAR A TRIPLE POINT}

In this section we consider interfacial properties of coexisting isotropic and nematic phases near the triple point in the bulk phase diagram of Fig. 1(b). We choose $\varepsilon:=\mu_{2}^{*}-\mu_{2}^{* \text { tri }}>0$ at $I-N_{2}$ coexistence as a measure for the undersaturation with respect to the chemical potential of the small platelets at the triple point $\mu_{1}^{* \text { tri }}=5.294376743251(1), \mu_{2}^{* \operatorname{tri}}=3.054579122021(1)$. Notice that for the present Zwanzig model the accuracy of the bulk coexistence data is higher than those one would be able to achieve for models of continuously rotating platelets because only a finite set of coexistence equations has to be solved instead of nonlinear integral equations. Hence, the undersaturation $\varepsilon$ can be defined rather precisely in the limit $\varepsilon \rightarrow 0$ within the present model. This is crucial for the subsequent wetting analysis and motivates the use of the Zwanzig approximation.

Fig. [5 shows the density profiles of the large and the small platelets for various values of $\varepsilon$. The density profiles exhibit a plateau region, signaling the onset of the formation of the still metastable $N_{1}$ phase. In the limit of $\varepsilon \rightarrow 0$, the local density of the plateau region agrees with the one of the bulk phase $N_{1}$ at the triple point, whereas the asymptotic densities at $z \rightarrow \pm \infty$ are the ones of the coexisting phases $I$ and $N_{2}$.

Fig. [6] shows the interface thickness $t$ as a function of
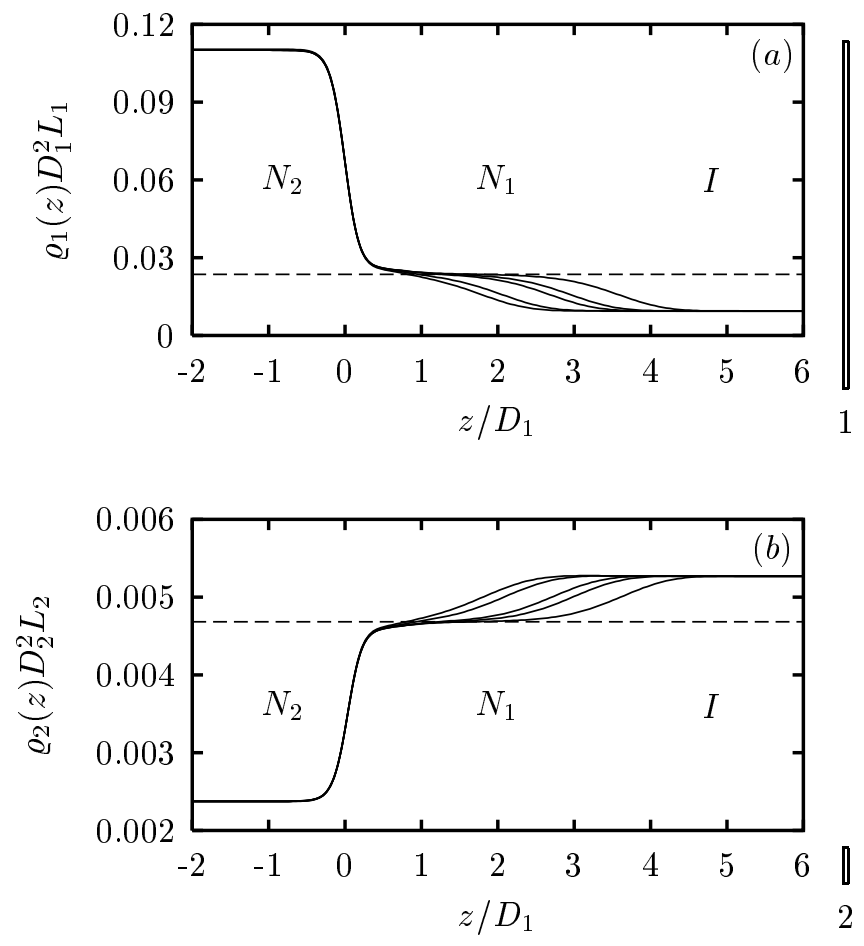

FIG. 5: Equilibrium density profiles of large (a) and small (b) thin platelets (see Fig. 1(b)) at various triple point undersaturations $\varepsilon:=\mu_{2}^{*}-\mu_{2}^{* \text { tri }} \in\left\{10^{-3}, 5 \times 10^{-4}, 10^{-4}, 5 \times 10^{-5}, 10^{-5}\right\}$ (from left to right) at $I-N_{2}$ coexistence. The position $z=0$ of the origin is chosen as the midpoint of the bulk densities of $N_{1}$ and $N_{2}$. The dashed lines represent the densities of the nematic phase $N_{1}$ at the triple point. The isotropic phase $I$ is at $z \rightarrow \infty$, and the nematic phase $N_{2}$ is at $z \rightarrow-\infty$.

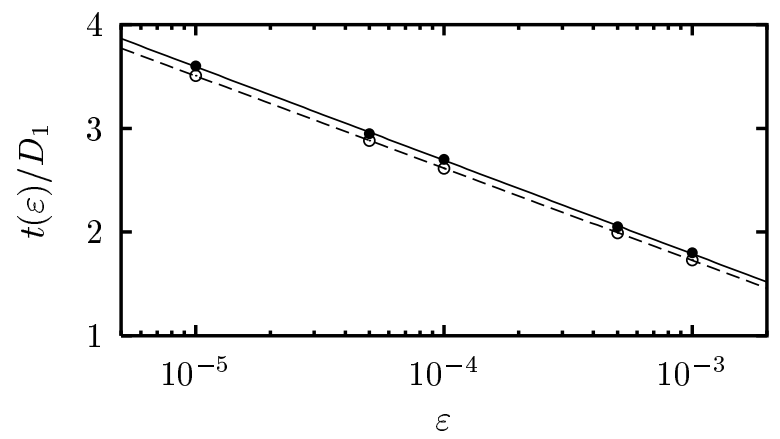

FIG. 6: Thickness $t$ of the $I-N_{2}$ interface as a function of the undersaturation $\varepsilon:=\mu_{2}^{*}-\mu_{2}^{* \operatorname{tri}}$ from the triple point (see Figs. 1(b) and 5). The solid and the dashed line are calculated according to the inflection point and the midpoint method, respectively, as described in the text. The correlation length $\xi=0.389(3) D_{1}$ is determined from the slope of the logarithmic growth of $t$ for $\varepsilon \rightarrow 0$.

the undersaturation $\varepsilon$. There are various possible definitions for the interface thickness. We have used the following two measures:

- The inflection point method: The interface thickness is given by the distance between the outermost 
inflection points of the density profiles.

- The midpoint method: The distance between the midpoints of the density profiles for the $I-N_{1}$ and the $N_{1}-N_{2}$ interface defines the interfacial thickness.

Upon approaching the triple point, we find that $t$ diverges for $\varepsilon \rightarrow 0$ as $t \simeq-\xi \ln \varepsilon+t_{1}$ with $\xi=0.392(4) D_{1}$, $t_{1}=-0.92(4) D_{1}$ for the inflection point method and $\xi=0.386(2) D_{1}, t_{1}=-0.94(2) D_{1}$ for the midpoint method (see Fig. 6). The logarithmic divergence of $t$ is consistent with complete wetting of the $I-N_{2}$ interface by a $N_{1}$ film at the triple point in the absence of algebraically decaying interaction potentials 33. Moreover, we have confirmed that the value of $\xi$ determined from the thickness of the interface is in agreement with the correlation length of the wetting phase $N_{1}$ at the triple point. The latter has been obtained from the asymptotic exponential decay of the density profiles from $I-N_{1}$ or $N_{1}-N_{2}$ interfaces towards their $N_{1}$ bulk values at the triple point.

The importance of interface fluctuations is determined by the so-called wetting parameter 34]

$$
\omega=\frac{k_{B} T}{4 \pi \gamma_{I N_{2}} \xi^{2}}
$$

which for the present system takes a value of order unity at the triple point. Fluctuations are therefore neither negligible nor dominant.

In order to validate complete triple point wetting by an alternative method, the vanishing of the dihedral angle $\vartheta_{N_{1}}$ of a lense of phase $N_{1}$ at the triple point has been checked using the relation

$$
\cos \vartheta_{N_{1}}=\frac{\left(\gamma_{I N_{2}}^{\mathrm{tri}}\right)^{2}-\left(\gamma_{I N_{1}}^{\mathrm{tri}}\right)^{2}-\left(\gamma_{N_{1} N_{2}}^{\mathrm{tri}}\right)^{2}}{2 \gamma_{I N_{1}}^{\mathrm{tri}} \gamma_{N_{1} N_{2}}^{\mathrm{tri}}}
$$

The interfacial tensions $\gamma_{I N_{1}}^{\mathrm{tri}}=0.05003056658 k_{B} T / D_{1}^{2}$ and $\gamma_{N_{1} N_{2}}^{\mathrm{tri}}=0.05600483075 k_{B} T / D_{1}^{2}$ are obtained by approaching the triple point along the $I-N_{1}$ and the $N_{1}-N_{2}$ coexistence curves, respectively, from below in terms of the chemical potential of the small platelets $\mu_{2}^{*}$ (see Fig. 1(b)).

Recently, complete wetting of an $I-N_{2}$ interface by a $N_{1}$ film has been predicted for a binary mixture of long thin and thick rods $(L>D)$ with $L_{1}=L_{2}, D_{1} / D_{2} \geq 4$ [35, 36]. The ratio $\xi / L_{1}$ in those systems is of the same order of magnitude as the value $\xi / D_{1}=0.389(3)$ we have obtained for a binary mixture of large and small platelets $(L<D)$ with $L_{1}=L_{2}, D_{1} / D_{2}=10$.

At the emerging $I-N_{1}$ interface, the orientational order parameter profiles $s_{1}$ and $s_{2}$ exhibit slightly negative minima indicating the depletion of platelets with orientation parallel to the interface. The absolute values of these minima are two orders of magnitude smaller for $s_{2}$ than for $s_{1}$.

\section{SUMMARY}

We have studied bulk and interfacial properties of binary hard-platelet fluids using density-functional theory. The platelets form square parallelepipeds with orientations restricted to three mutually perpendicular directions. A fundamental measure theory is used to derive the excess free energy functional. The grand potential functional is minimized numerically and phase diagrams, density profiles, orientational order parameter profiles, and surface tensions are determined leading to the following main results:

1. The bulk phase diagrams for two representative examples of binary hard-platelet fluids (Fig. 11) involve an isotropic and one or two nematic phases of different concentration as well as a columnar phase.

2. For a binary platelet mixture of large thin and small thick platelets, the density profiles of thick platelets at the isotropic-nematic interface exhibit oscillations (Fig. 22). A density inversion for the thick platelets is found. Near the thermodynamic state marked as $b$ in Fig. 2] the densities of the thick platelets in the isotropic and the nematic phase are nearly identical. The interfacial tension for platelets with their mean bulk orientation parallel to the interface is smaller than for the corresponding perpendicular configuration. The bidisperse mixtures have significantly larger interfacial tensions than the corresponding monodisperse fluids (Fig. 3).

3. Both the large platelet and the small platelet density profiles exhibit pronounced oscillations on one side of the nematic-nematic interface, provided the chemical potentials are sufficiently high (Fig. 4, state $d)$. Upon approaching the lower critical point of nematic-nematic demixing the oscillations vanish and the interface broadens (Fig. 4 state $e$ ).

4. Just above the isotropic-nematic-nematic triple point shown in Fig. 1(b), the $I-N_{2}$ interfacial profiles thicken due to the formation of the incipient nematic phase $N_{1}$ at this interface (Fig. 5). Fig. 6] demonstrates that the thickness of the wetting film diverges logarithmically upon approaching the triple point. Complete triple point wetting is confirmed explicitly by observing the vanishing of the dihedral angle at the triple point.

\section{Acknowledgments}

The authors thank R. van Roij for useful discussions. 
[1] T.G. Mason, Phys. Rev. E 66, 060402(R) (2002).

[2] G.C. Maitland, Curr. Opin. Colloid Interface Sci. 5, 301 (2000).

[3] R.J. Bushby and O.R. Lozman, Curr. Opin. Colloid Interface Sci. 7, 343 (2002).

[4] L. Onsager, Phys. Rev. 62, 558 (1942).

[5] L. Onsager, Ann. N. Y. Acad. Sci. 51, 627 (1949).

[6] R. Eppenga and D. Frenkel, Mol. Phys. 52, 1303 (1984).

[7] J.A.C. Veerman and D. Frenkel, Phys. Rev. A 45, 5632 (1992).

[8] A. Casey, P. Harrowell, J. Chem. Phys. 103, 6143 (1995).

[9] A. Mourchid, A. Delville, J. Lambard, E. Lécolier, and P. Levitz, Langmuir 11, 1942 (1995).

[10] J.-C.P. Gabriel, C. Sanchez, and P. Davidson, J. Phys. Chem. 100, 11139 (1996).

[11] A.B.D. Brown, S.M. Clarke, A.R. Rennie, Langmuir 14, 3129 (1998).

[12] F.M. van der Kooij and H.N.W. Lekkerkerker, J. Phys. Chem. B 102, 7829 (1998).

[13] F.M. van der Kooij, D. van der Beek, and H.N.W. Lekkerkerker, J. Phys. Chem. B 105, 1696 (2001).

[14] D. van der Beek and H.N.W. Lekkerkerker, Europhys. Lett. 61, 702 (2003).

[15] M.A. Bates and D. Frenkel, J. Chem. Phys. 110, 6553 (1999).

[16] H.H. Wensink, G.J. Vroege, and H.N.W. Lekkerkerker, J. Phys. Chem. B 105, 10610, (2001).

[17] L. Harnau, D. Rowan, and J.-P. Hansen, J. Chem. Phys. 117, 11359 (2002).

[18] Y. Martínez-Ratón and J.A. Cuesta, J. Chem. Phys. 118, 10164 (2003).

[19] L. Harnau and S. Dietrich, Phys. Rev. E 65, 021505 (2002).

[20] L. Harnau and S. Dietrich, Phys. Rev. E 66, 051702 (2002).

[21] R. Zwanzig, J. Chem. Phys. 39, 1714 (1963).
[22] J.A. Cuesta and Y. Martínez-Ratón, Phys. Rev. Lett. 78, 3681 (1997).

[23] J.A. Cuesta and Y. Martínez-Ratón, J. Chem. Phys. 107, 6379 (1997).

[24] J.A. Cuesta and R.P. Sear, Eur. Phys. J. B 8, 233 (1999).

[25] Y. Martínez-Ratón, J.A. Cuesta, R. van Roij, and B. Mulder, in New approaches to problems in liquid state theory, edited by C. Caccamo, J.-P. Hansen, and G. Stell (Kluwer Academic, Dordrecht, 1999), NATO science series Vol. C 529, p. 139.

[26] H.N.W. Lekkerkerker, Ph. Coulon, R. van der Haegen, J. Chem. Phys. 80, 3427 (1984).

[27] K. Shundyak and R. van Roij, J. Phys.: Condens. Matter 13, 4789 (2001).

[28] M.E. Fisher and B. Widom, J. Chem. Phys. 50, 3756 (1969).

[29] R. Evans, J.R. Henderson, D.C. Hoyle, A.O. Parry, and Z.A. Sabeur, Mol. Phys. 80, 755 (1993).

[30] R. Evans, R.J.F. Leote de Carvalho, J.R. Henderson, and D.C. Hoyle, J. Chem. Phys. 100, 591 (1994).

[31] A.J. Archer and R. Evans, Phys. Rev. E 64, 041501 (2001).

[32] J.M. Brader, R. Evans, M. Schmidt, and H. Löwen, J. Phys.: Condens. Matter 14, L1 (2002).

[33] S. Dietrich, in Phase Transitions and Critical Phenomena, edited by C. Domb and J.L. Lebowitz (Academic, London, 1988), Vol. 12, p. 1.

[34] M. Schick, in Les Houches, Session XLVIII, 1988 - Liquides aux interfaces / Liquids at interfaces, edited by J. Charvolin, J.F. Joanny, and J. Zinn-Justin (NorthHolland, Amsterdam, 1990), p. 415.

[35] K. Shundyak and R. van Roij, Phys. Rev. Lett. 88, 205501 (2002).

[36] K. Shundyak and R. van Roij, cond-mat/0309414 (2003). 